Supplement of Biogeosciences, 17, 281-304, 2020

https://doi.org/10.5194/bg-17-281-2020-supplement

(c) Author(s) 2020. This work is distributed under

the Creative Commons Attribution 4.0 License.

(c) (1)

Supplement of

\title{
Current, steady-state and historical weathering rates of base cations at two forest sites in northern and southern Sweden: a comparison of three methods
}

Sophie Casetou-Gustafson et al.

Correspondence to: Sophie Casetou-Gustafson (sophie.casetou@slu.se)

The copyright of individual parts of the supplement might differ from the CC BY 4.0 License. 
Figure S1. Zirkonium $(\mathrm{Zr})$ to base cation $(\mathrm{Ca}, \mathrm{Mg}, \mathrm{K}, \mathrm{Na})$ ratios by concentration at different soil layers at Flakaliden (left) and Asa (right).

\section{Flakaliden}
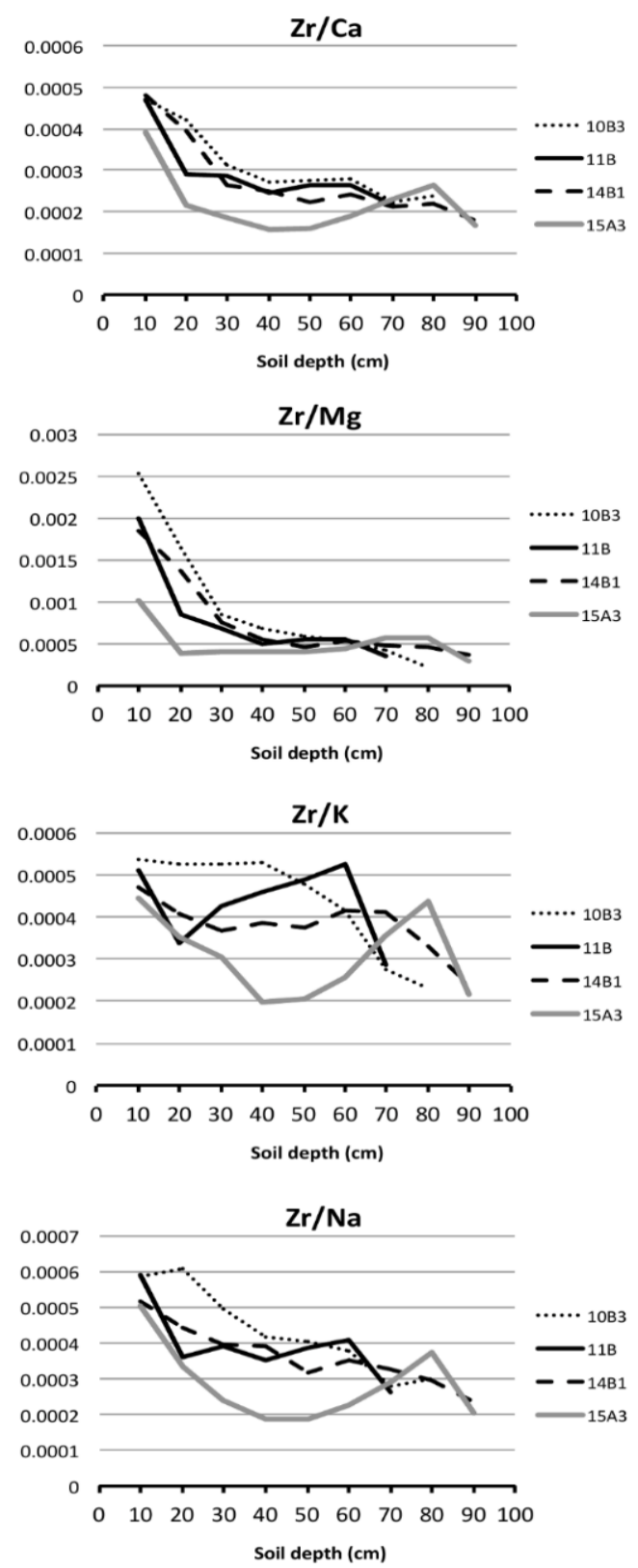

Asa
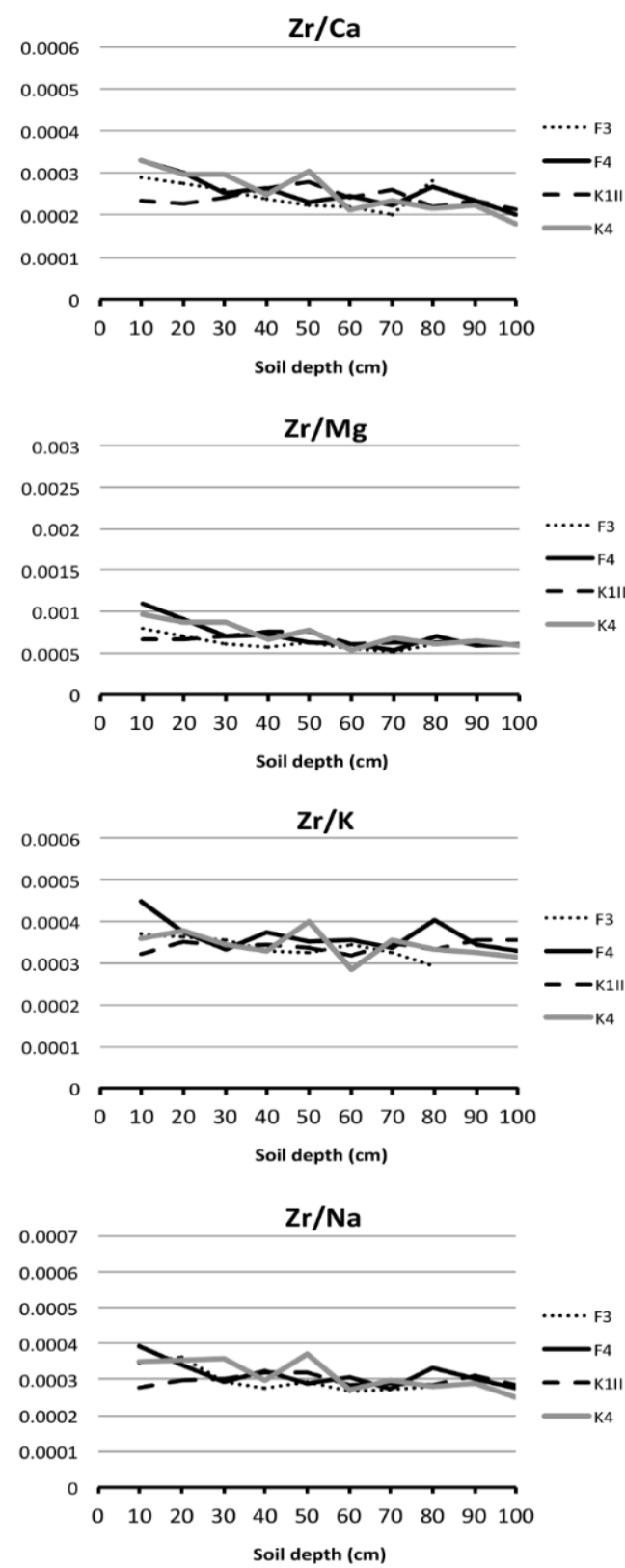
Figure S2. Mean and SE of BC ions $(\mathrm{mg} / \mathrm{l})$ in soil water sampled at $50 \mathrm{~cm}$ depth in the soil of the four control plots at Asa during 1990-2004 and at Flakaliden during 1988-2004.

$\mathrm{BC}(\mathrm{mg} / \mathrm{l})$ in soil water at Flakaliden

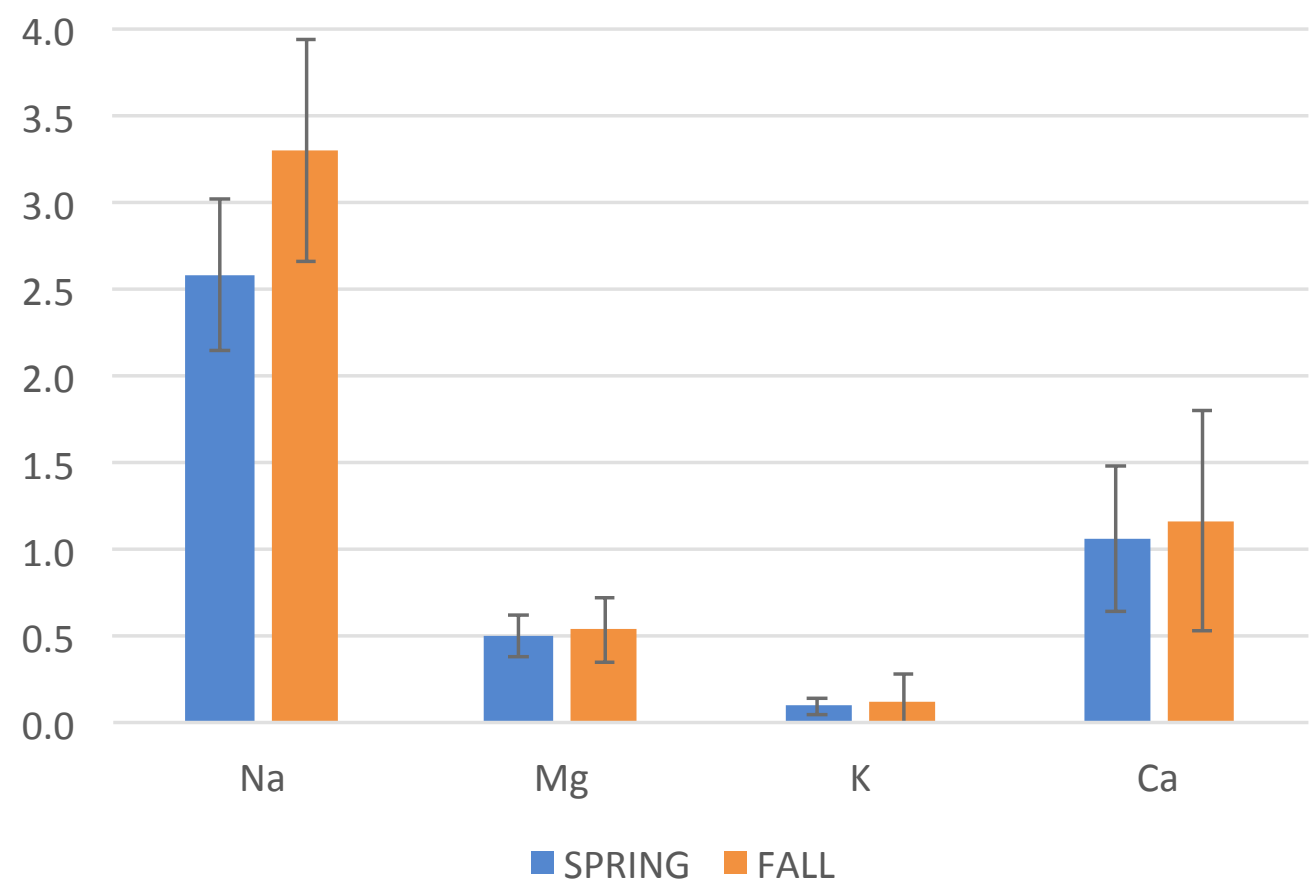

$\mathrm{BC}(\mathrm{mg} / \mathrm{l})$ in soil water at Asa

4.0

3.5

3.0

2.5

2.0

1.5

1.0

0.5

0.0

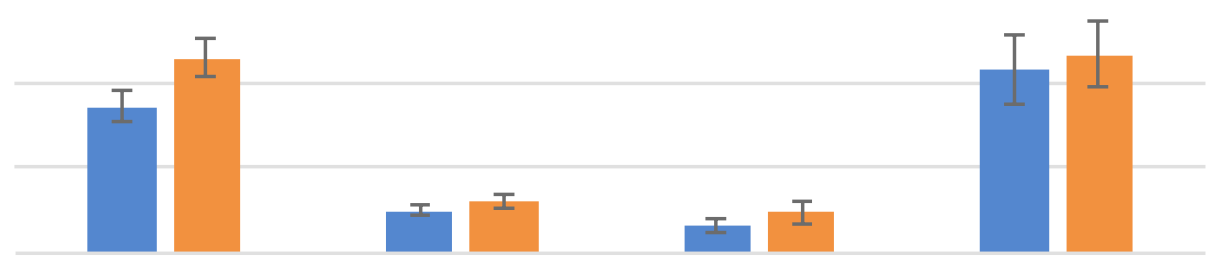

$\mathrm{Na}$

$\mathrm{Mg}$

K

$\mathrm{Ca}$

SPRING FALL 
Figure S3. Monthly mean and SD drainage $(\mathrm{mm})$ at $50 \mathrm{~cm}$ depth in the soil of control plots at Asa during 1990-2004 and at Flakaliden during 1988-2004.

Drainage at Flakaliden, $\mathrm{mm} / \mathrm{month}$ 120

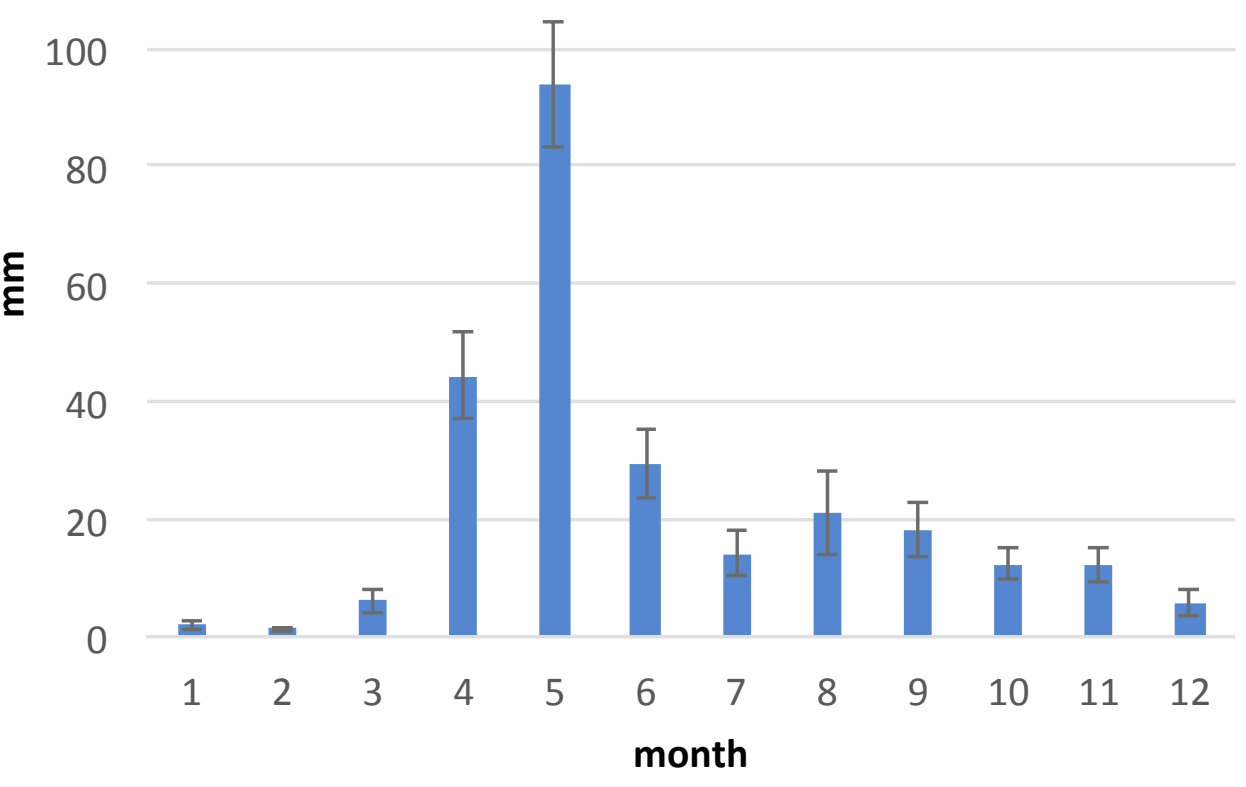

Drainage at Asa, $\mathrm{mm} /$ month

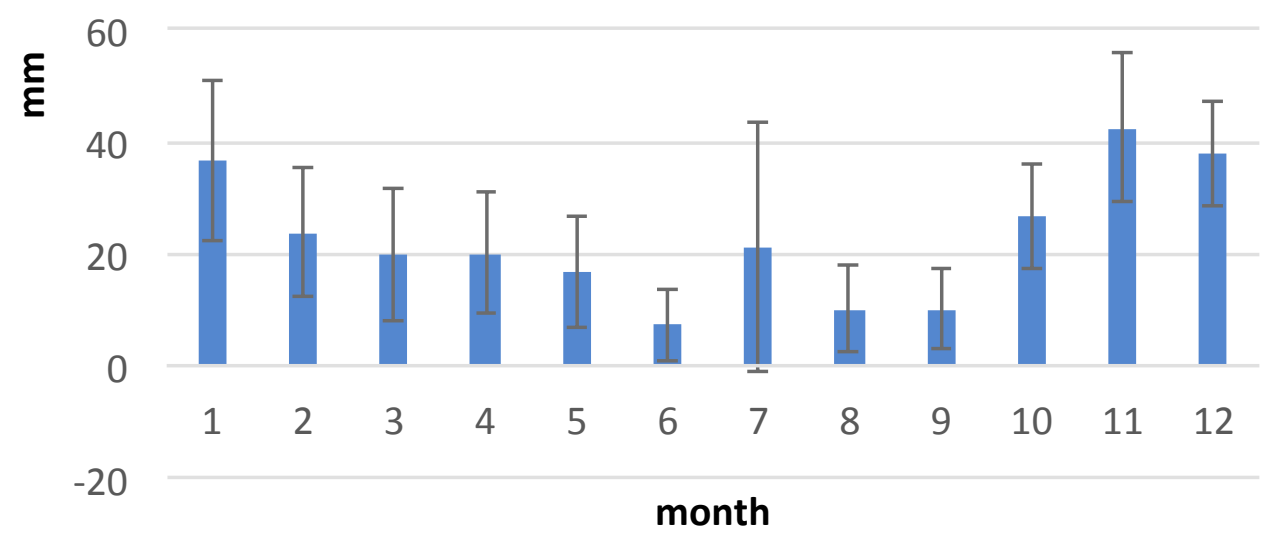




\section{Supplement $4 a$}

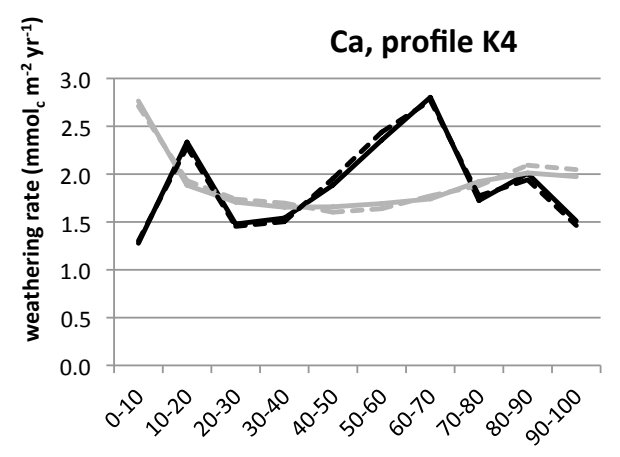

Soil depth (cm)
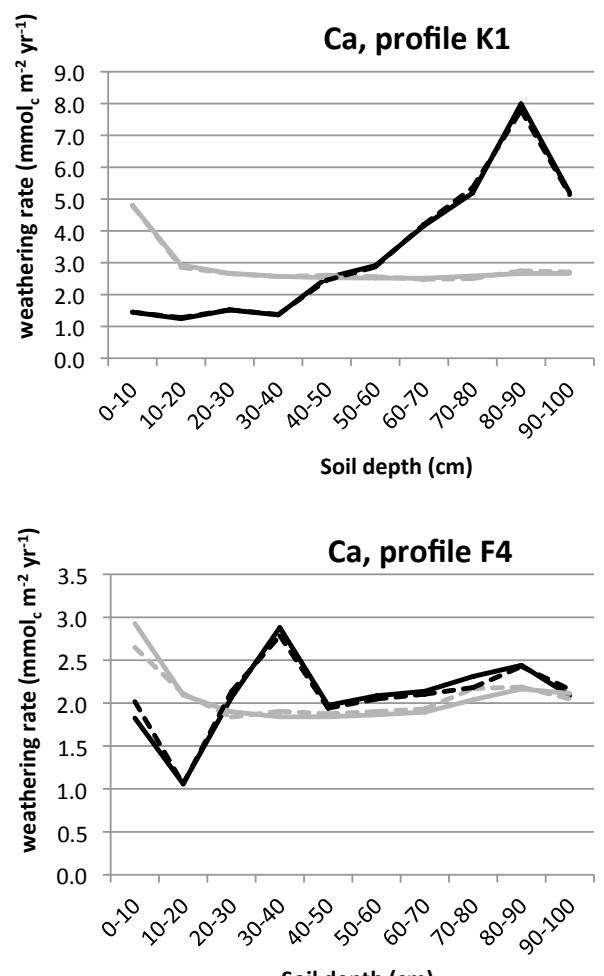

Soil depth (cm)

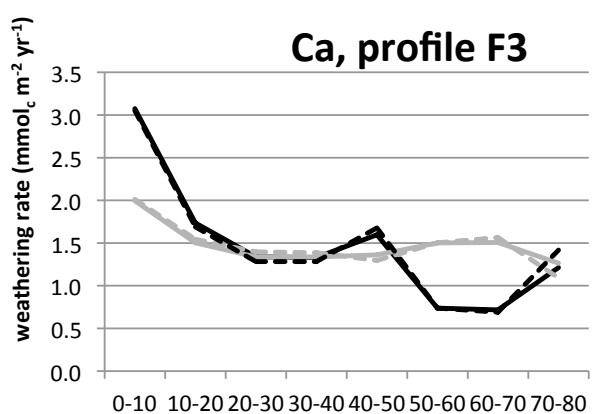

Soil depth (cm)
Asa
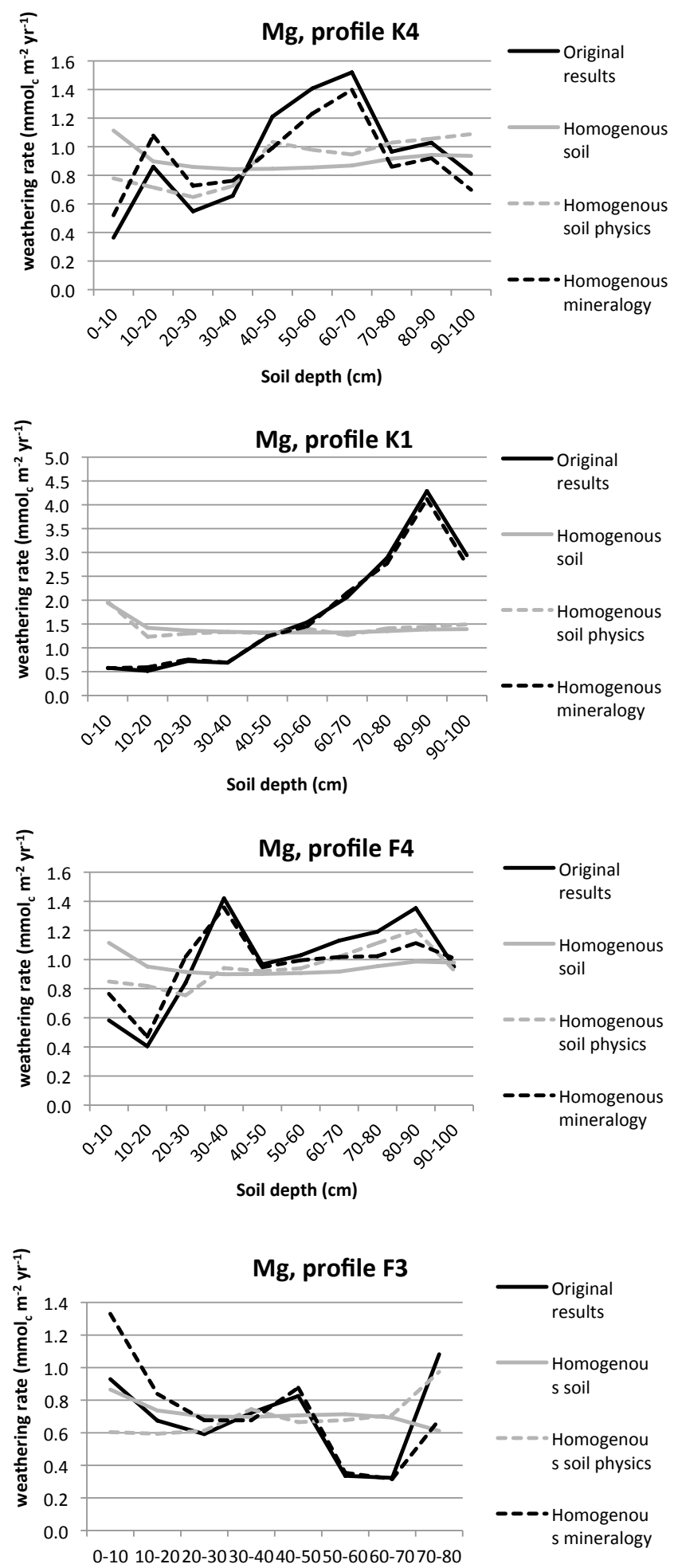

Soil depth (cm) 


\section{Supplement 4b}

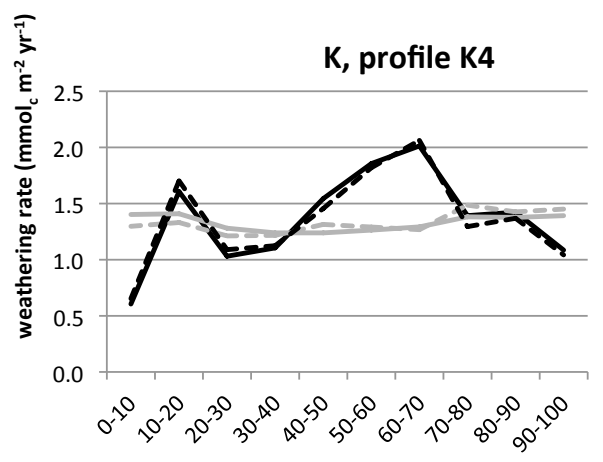

Soil depth $(\mathrm{cm})$

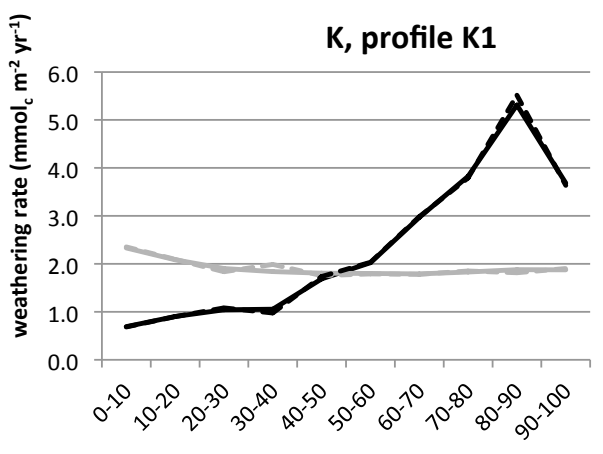

Soil depth (cm)

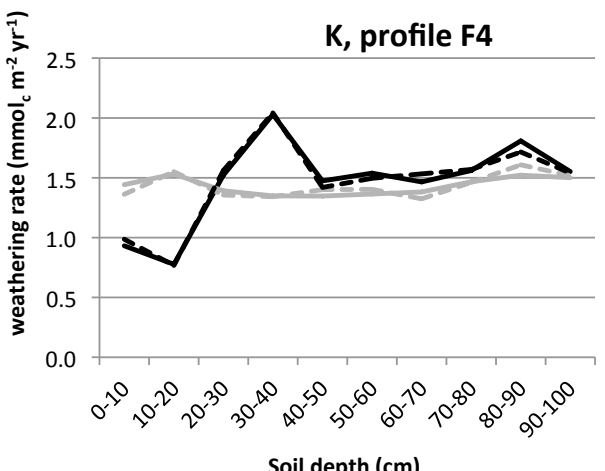

Soil depth (cm)

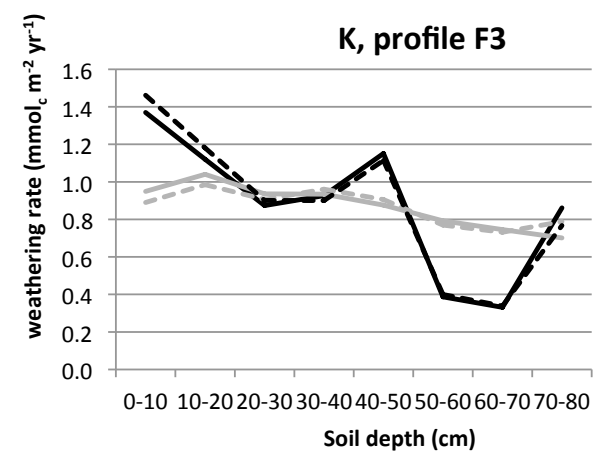

Asa
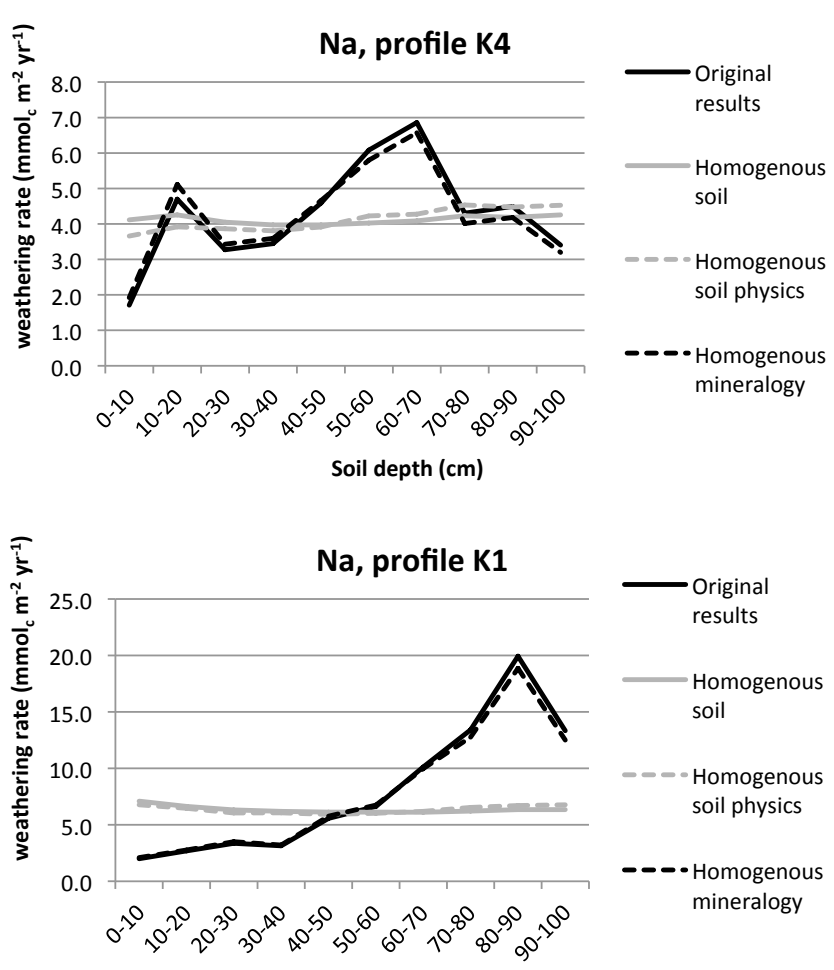

Soil depth (cm)
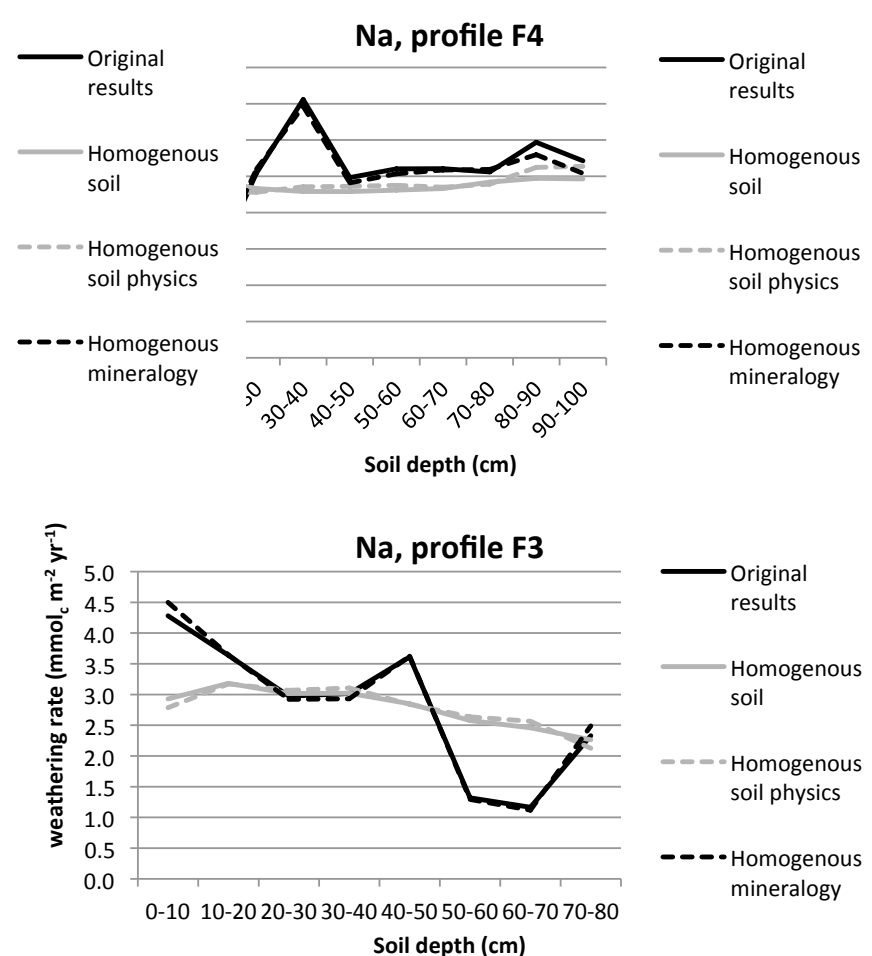


\section{Supplement 5a}

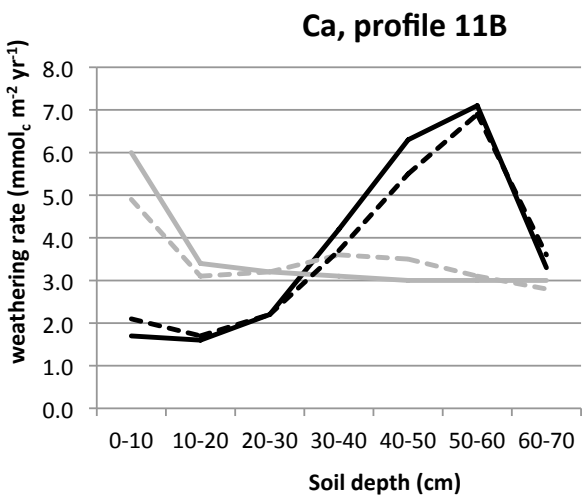

Ca, profile 14B

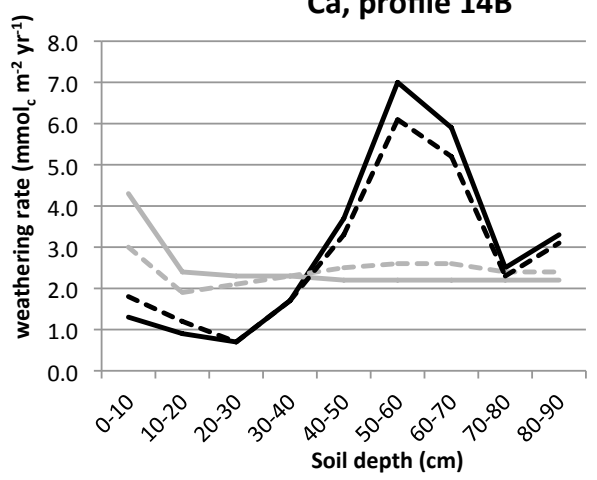

Ca, profile 15A

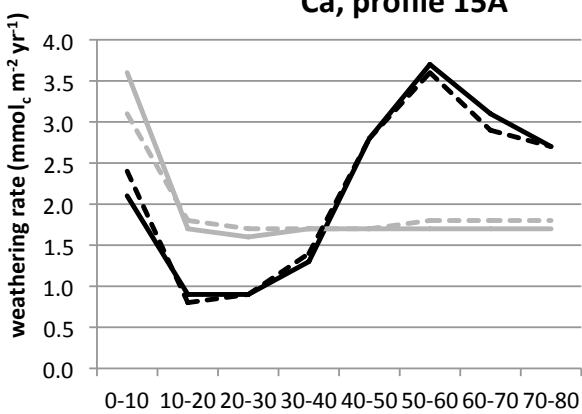

Soil depth $(\mathrm{cm})$

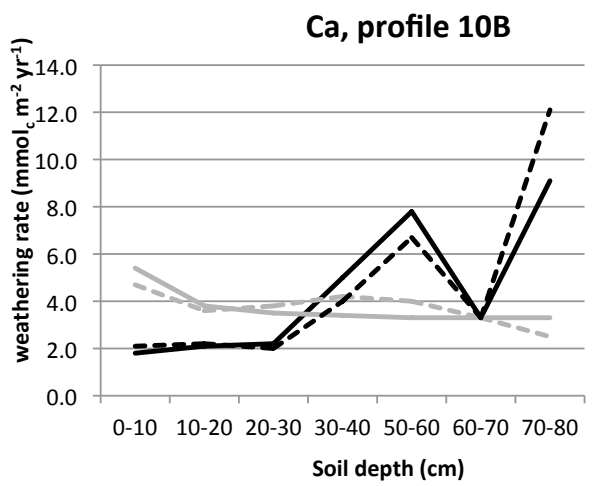

Flakaliden
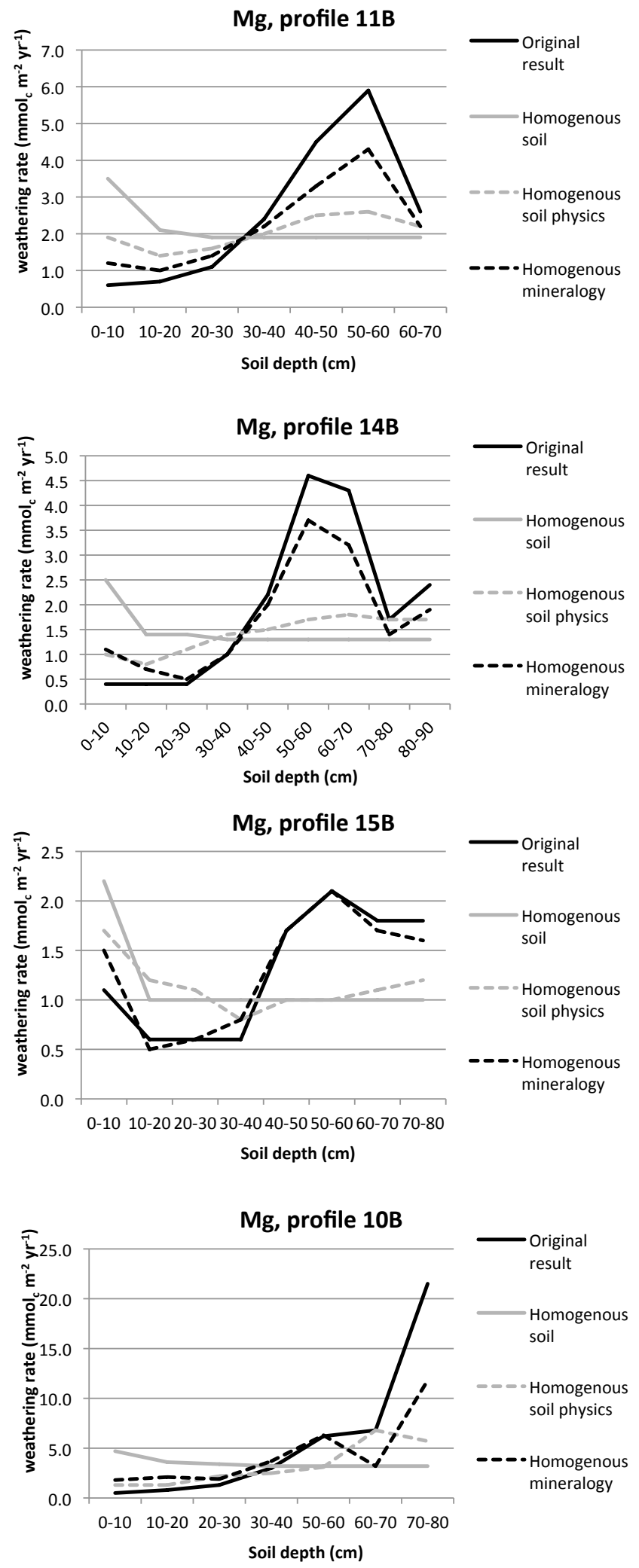


\section{Supplement 5b}
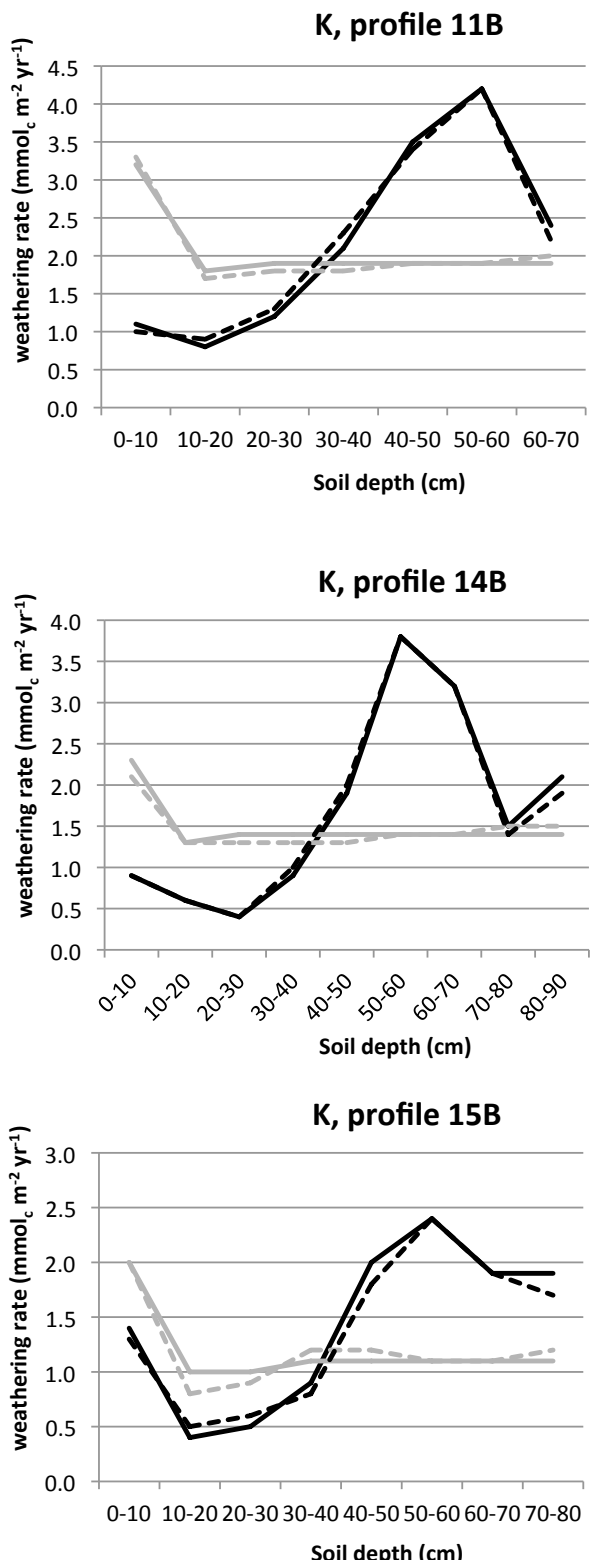

Soil depth (cm)

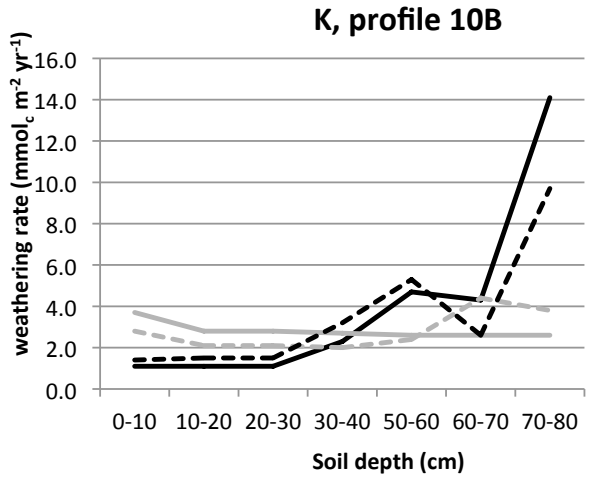

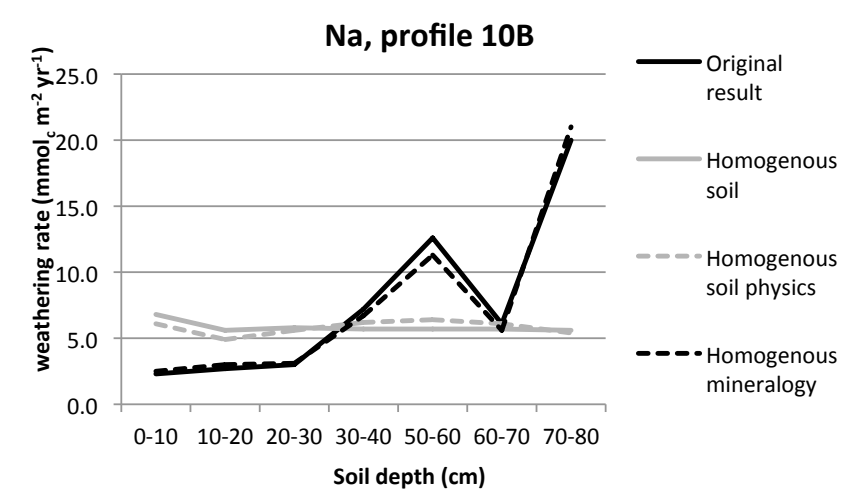

$\mathrm{Na}$, profile 11B
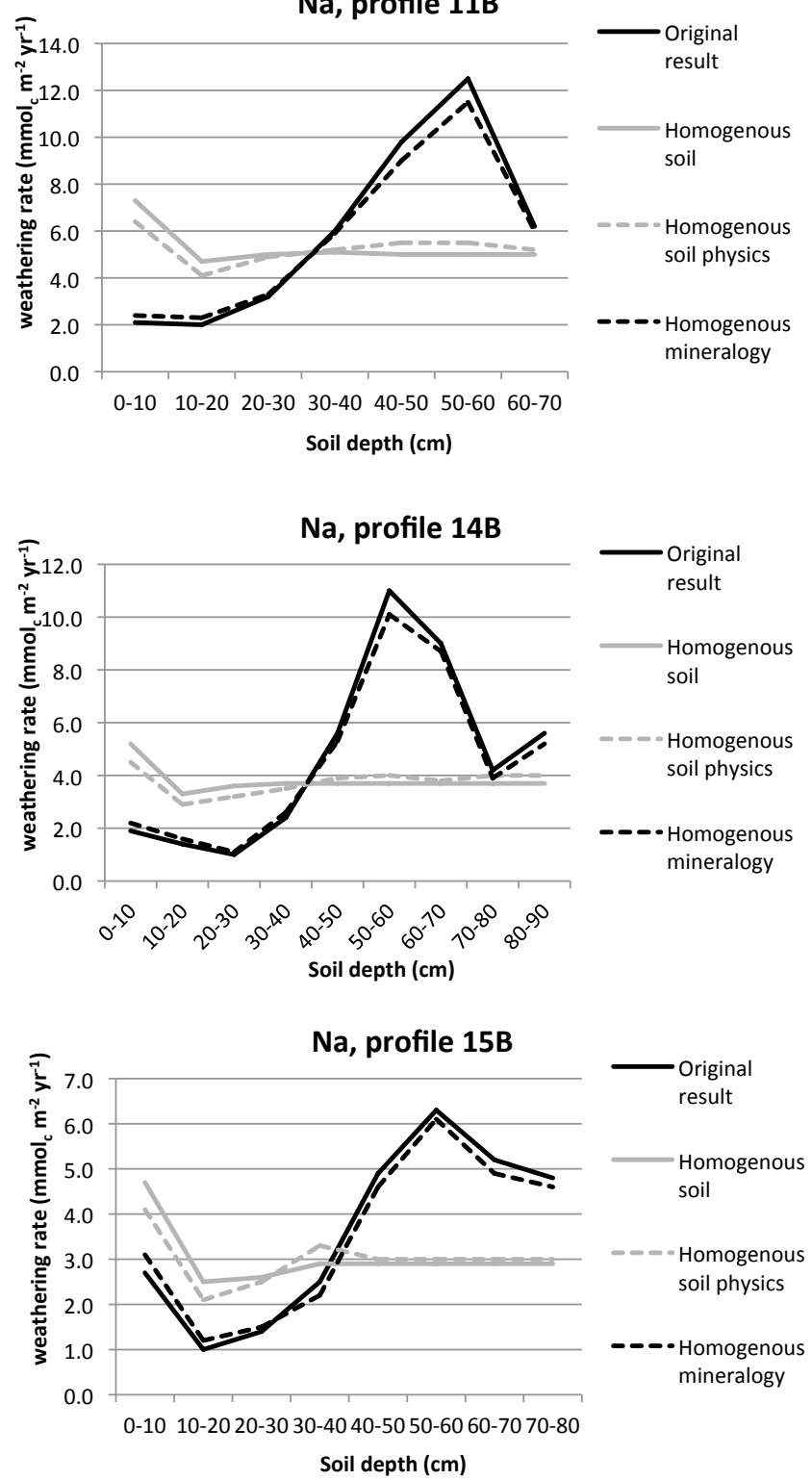
Figure S6. Weathering rate (mmolc $\mathrm{m}-2 \mathrm{yr}-1)$ of $\mathrm{Ca}(\mathrm{a}), \mathrm{K}(\mathrm{b}), \mathrm{Mg}(\mathrm{c})$ and $\mathrm{Na}(\mathrm{d})$ as a function of exposed soil surface area ( $\mathrm{m} 2 \mathrm{~m}-3)$ at Asa (white circles) and Flakaliden (grey circles).
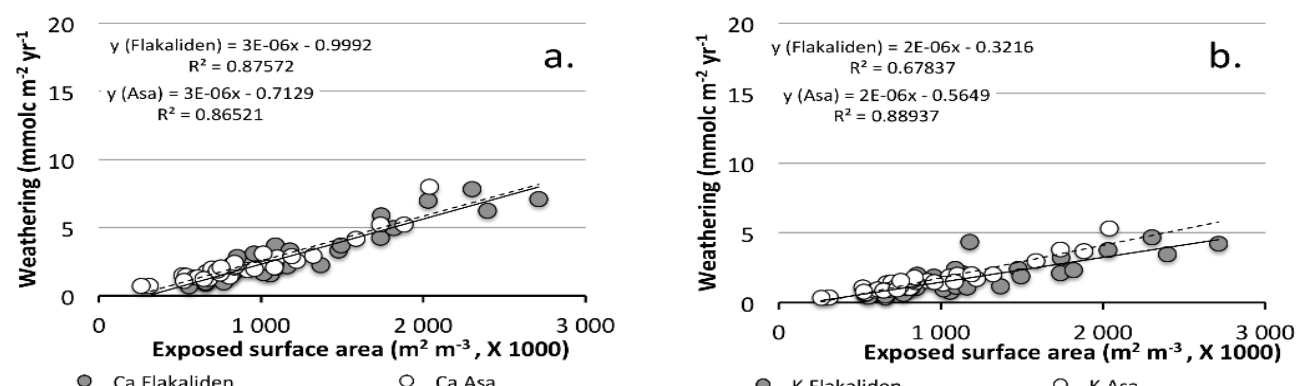

- Ca Flakaliden $O$ Ca Asa

- K Flakaliden

$$
\text { - K Asa }
$$
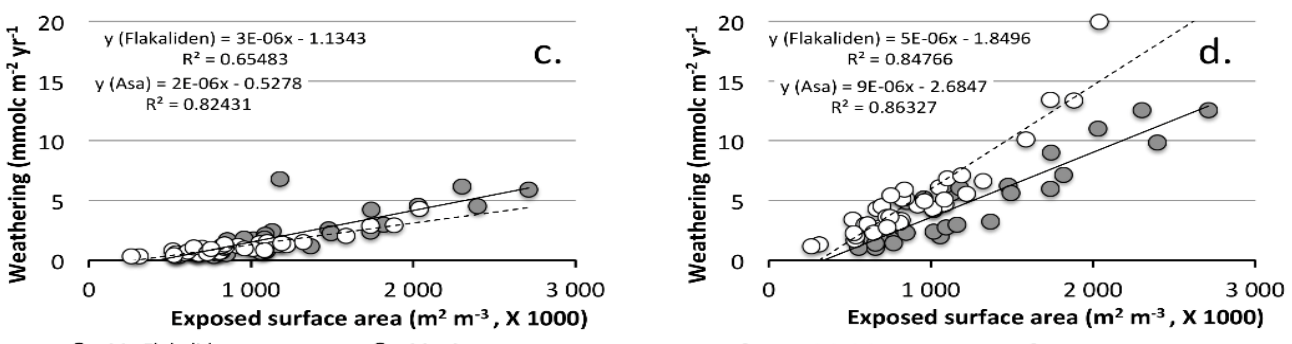

Mg Flakaliden 
Figure S7. Weathering rate (mmolc $\mathrm{m}-2 \mathrm{yr}-1$ ) of $\mathrm{Ca}(\mathrm{a}), \mathrm{K}(\mathrm{b}), \mathrm{Mg}(\mathrm{c})$ and $\mathrm{Na}(\mathrm{d})$ as a function of bulk density (g L-1) at Asa (white circles) and Flakaliden (grey circles).
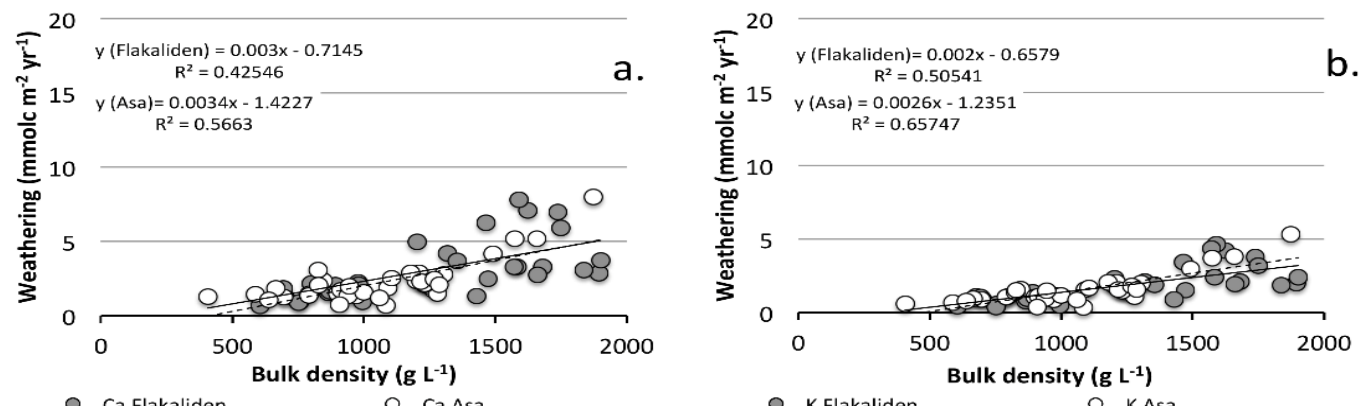

- Ca Flakaliden

O Ca Asa

○ K Flakaliden

O KAsa

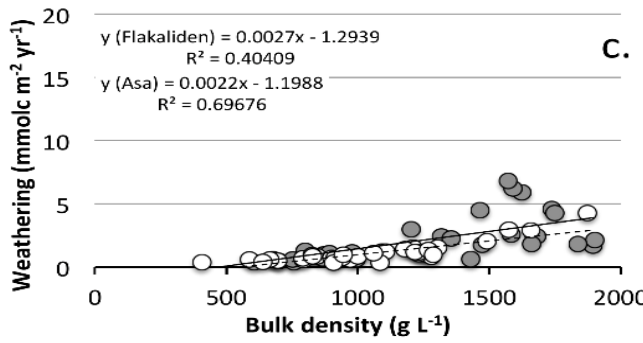

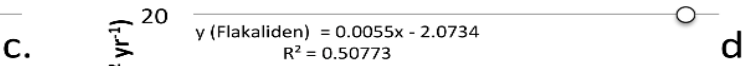

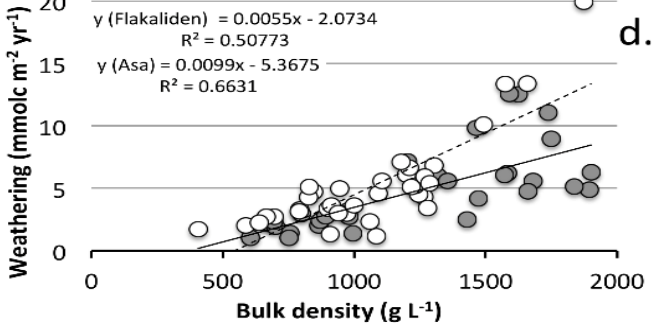

- Mg Flakaliden

$\circ$ Mg Flakaliden $\quad \circ$ Mg Asa

- Na Flakaliden

○ Na Asa . 
Table S1. XRPD Mineralogical composition (weight \%) of the fine-earth ( $<2 \mathrm{~mm}$ ) fraction of soils from Asa (upper table) and Flakaliden (lower table). Dioct.=dioctahedral phyllosillicates; Trioct.=trioctahedral phyllosilicates.

\begin{tabular}{|c|c|c|c|c|c|c|c|c|c|c|c|c|c|c|c|c|c|c|c|c|}
\hline Site & Plot & Depth & Quartz & $\begin{array}{c}\text { K- } \\
\text { feldspar }\end{array}$ & $\begin{array}{l}\begin{array}{c}\text { Plagio- } \\
\text { clase }\end{array} \\
\end{array}$ & $\begin{array}{c}\text { Amphi- } \\
\text { bole }\end{array}$ & $\begin{array}{l}\text { Epid- } \\
\text { ote }\end{array}$ & $\begin{array}{l}\text { Anat- } \\
\text { ase }\end{array}$ & $\begin{array}{c}\text { IIme- } \\
\text { nite }\end{array}$ & $\begin{array}{c}\text { Hema- } \\
\text { tite }\end{array}$ & $\begin{array}{c}\text { Chlorite } \\
\text { trioct. }\end{array}$ & $\begin{array}{l}\text { Mica } \\
\text { dioct. }\end{array}$ & $\begin{array}{l}\text { Mica } \\
\text { trioct. }\end{array}$ & $\begin{array}{l}\text { Verm. } \\
\text { dioct. }\end{array}$ & $\begin{array}{l}\text { Verm. } \\
\text { trioct. }\end{array}$ & $\begin{array}{l}\text { Hydro- } \\
\text { biotite }\end{array}$ & $\begin{array}{c}\text { Kaoli- } \\
\text { nite }\end{array}$ & $\begin{array}{l}\mathrm{m} . / \\
\text { Allo. }\end{array}$ & $\begin{array}{c}\text { Ferri- } \\
\text { hydrite }\end{array}$ & Total \\
\hline Asa & K1 & $0-10$ & 43.6 & 15.3 & 25.1 & 2.6 & 3.2 & 0.0 & 0.6 & 0.9 & 1.0 & 3.3 & 0.0 & 0.9 & 0.2 & 0.7 & 0.0 & 1.8 & 0.8 & 100 \\
\hline Asa & K1 & $10-20$ & 44.0 & 15.3 & 25.7 & 2.4 & 3.0 & 0.0 & 0.6 & 1.0 & 0.9 & 2.9 & 0.2 & 1.2 & 0.2 & 0.2 & 0.0 & 1.6 & 1.0 & 100 \\
\hline Asa & K1 & $20-30$ & 44.2 & 14.4 & 25.2 & 2.5 & 3.2 & 0.0 & 0.5 & 0.9 & 1.0 & 3.5 & 0.0 & 1.1 & 0.2 & 0.5 & 0.0 & 1.9 & 0.8 & 100 \\
\hline Asa & K1 & $30-40$ & 42.6 & 16.4 & 25.8 & 2.4 & 3.0 & 0.0 & 0.6 & 0.9 & 1.1 & 3.1 & 0.3 & 1.4 & 0.2 & 0.6 & 0.0 & 0.9 & 0.9 & 100 \\
\hline Asa & K1 & $40-50$ & 44.7 & 14.8 & 25.4 & 2.6 & 3.2 & 0.0 & 0.6 & 1.0 & 1.2 & 2.8 & 0.1 & 1.4 & 0.1 & 0.4 & 0.0 & 0.0 & 1.5 & 100 \\
\hline Asa & K1 & $50-60$ & 44.1 & 15.1 & 25.9 & 2.5 & 3.2 & 0.0 & 0.6 & 0.9 & 1.2 & 3.0 & 0.0 & 1.1 & 0.1 & 0.9 & 0.0 & 0.0 & 1.3 & 100 \\
\hline Asa & K1 & $60-70$ & 44.0 & 14.9 & 26.7 & 2.2 & 3.4 & 0.0 & 0.6 & 0.9 & 1.5 & 3.1 & 0.3 & 0.6 & 0.1 & 0.4 & 0.0 & 0.2 & 1.2 & 100 \\
\hline Asa & K1 & $70-80$ & 43.8 & 15.0 & 27.7 & 2.3 & 3.1 & 0.0 & 0.5 & 0.8 & 1.2 & 3.5 & 0.0 & 0.2 & 0.2 & 1.0 & 0.0 & 0.0 & 0.6 & 100 \\
\hline Asa & K1 & $80-90$ & 43.8 & 14.6 & 27.9 & 2.4 & 3.5 & 0.0 & 0.6 & 0.8 & 1.1 & 2.8 & 0.0 & 0.4 & 0.1 & 0.9 & 0.0 & 0.0 & 1.0 & 100 \\
\hline Asa & K1 & $90-100$ & 42.7 & 15.2 & 28.1 & 2.6 & 3.3 & 0.0 & 0.5 & 0.9 & 1.3 & 3.2 & 0.1 & 0.1 & 0.1 & 0.7 & 0.0 & 0.0 & 1.1 & 100 \\
\hline Asa & K4 & $0-10$ & 45.6 & 15.2 & 23.5 & 2.2 & 3.0 & 0.0 & 0.6 & 0.9 & 0.6 & 2.5 & 0.1 & 2.0 & 0.2 & 0.0 & 0.0 & 1.6 & 1.9 & 100 \\
\hline Asa & K4 & $10-20$ & 45.5 & 15.2 & 24.3 & 2.4 & 3.1 & 0.1 & 0.6 & 1.0 & 0.6 & 2.2 & 0.2 & 2.2 & 0.2 & 0.0 & 0.0 & 1.0 & 1.4 & 100 \\
\hline Asa & K4 & $20-30$ & 44.8 & 15.4 & 25.2 & 2.0 & 3.1 & 0.0 & 0.5 & 0.9 & 0.9 & 2.1 & 0.2 & 2.3 & 0.2 & 0.2 & 0.0 & 0.0 & 2.1 & 100 \\
\hline Asa & K4 & $30-40$ & 43.4 & 15.5 & 25.3 & 2.5 & 3.0 & 0.1 & 0.5 & 0.9 & 1.0 & 3.2 & 0.1 & 1.8 & 0.1 & 0.1 & 0.0 & 0.8 & 1.8 & 100 \\
\hline Asa & K4 & $40-50$ & 41.7 & 15.8 & 25.9 & 2.5 & 3.0 & 0.0 & 0.5 & 0.8 & 1.3 & 4.9 & 0.0 & 0.3 & 0.3 & 1.3 & 0.0 & 1.3 & 0.3 & 100 \\
\hline Asa & K4 & $50-60$ & 41.5 & 15.4 & 27.7 & 2.4 & 3.0 & 0.0 & 0.6 & 0.8 & 1.5 & 4.4 & 0.0 & 0.0 & 0.3 & 1.1 & 0.0 & 0.7 & 0.8 & 100 \\
\hline Asa & K4 & $60-70$ & 43.4 & 15.0 & 27.6 & 2.3 & 3.3 & 0.0 & 0.6 & 1.0 & 1.4 & 3.4 & 0.1 & 0.3 & 0.2 & 0.9 & 0.0 & 0.0 & 0.4 & 100 \\
\hline Asa & K4 & $70-80$ & 40.7 & 15.9 & 28.4 & 2.2 & 3.1 & 0.0 & 0.5 & 0.8 & 1.3 & 4.4 & 0.6 & 0.0 & 0.2 & 0.9 & 0.0 & 0.9 & 0.3 & 100 \\
\hline Asa & K4 & $80-90$ & 41.5 & 16.1 & 28.4 & 2.5 & 3.4 & 0.0 & 0.5 & 0.8 & 1.5 & 3.4 & 0.3 & 0.2 & 0.1 & 0.7 & 0.0 & 0.0 & 0.6 & 100 \\
\hline Asa & K4 & $90-100$ & 41.6 & 16.2 & 28.2 & 2.4 & 3.4 & 0.0 & 0.5 & 0.9 & 1.4 & 3.1 & 0.3 & 0.3 & 0.2 & 1.0 & 0.0 & 0.0 & 0.6 & 100 \\
\hline Asa & F3 & $0-10$ & 44.5 & 14.6 & 25.3 & 2.2 & 3.3 & 0.1 & 0.5 & 0.8 & 1.0 & 2.4 & 0.2 & 1.2 & 0.3 & 0.0 & 0.0 & 1.3 & 2.5 & 100 \\
\hline Asa & F3 & $10-20$ & 43.8 & 14.2 & 26.4 & 2.3 & 3.3 & 0.1 & 0.5 & 0.7 & 1.3 & 2.8 & 0.2 & 1.3 & 0.3 & 0.0 & 0.0 & 0.0 & 2.8 & 100 \\
\hline Asa & F3 & $20-30$ & 41.8 & 14.5 & 26.9 & 2.3 & 3.7 & 0.0 & 0.5 & 0.8 & 1.7 & 3.1 & 0.1 & 0.7 & 0.2 & 0.3 & 0.0 & 1.8 & 1.5 & 100 \\
\hline Asa & F3 & $30-40$ & 41.1 & 15.2 & 27.2 & 2.4 & 3.6 & 0.0 & 0.4 & 0.8 & 2.0 & 3.6 & 0.0 & 0.5 & 0.2 & 0.9 & 0.0 & 0.7 & 1.3 & 100 \\
\hline Asa & F3 & $40-50$ & 42.5 & 15.5 & 26.4 & 2.4 & 3.1 & 0.0 & 0.4 & 0.7 & 1.7 & 3.3 & 0.2 & 0.1 & 0.3 & 0.4 & 0.0 & 2.0 & 1.1 & 100 \\
\hline Asa & F3 & $50-60$ & 43.0 & 14.9 & 27.2 & 2.2 & 3.2 & 0.0 & 0.5 & 0.8 & 2.0 & 2.5 & 0.2 & 0.7 & 0.1 & 0.5 & 0.0 & 0.0 & 2.0 & 100 \\
\hline Asa & F3 & $60-70$ & 42.0 & 15.2 & 27.7 & 2.6 & 3.3 & 0.0 & 0.4 & 0.7 & 2.0 & 2.6 & 0.0 & 0.6 & 0.3 & 0.5 & 0.0 & 0.0 & 2.1 & 100 \\
\hline Asa & F3 & $70-90$ & 40.6 & 17.0 & 27.9 & 2.1 & 2.7 & 0.1 & 0.4 & 0.8 & 1.7 & 2.9 & 0.3 & 0.7 & 0.1 & 0.5 & 0.0 & 0.7 & 1.5 & 100 \\
\hline Asa & F4 & $0-10$ & 46.7 & 14.7 & 24.0 & 1.9 & 2.8 & 0.1 & 0.5 & 0.8 & 0.7 & 2.9 & 0.0 & 1.1 & 0.3 & 0.3 & 0.0 & 1.9 & 1.3 & 100 \\
\hline Asa & F4 & $10-20$ & 44.9 & 15.9 & 25.0 & 2.1 & 3.2 & 0.0 & 0.5 & 0.9 & 0.9 & 1.8 & 0.3 & 1.7 & 0.2 & 0.2 & 0.0 & 0.4 & 2.1 & 100 \\
\hline Asa & F4 & $20-30$ & 44.8 & 15.0 & 26.4 & 1.9 & 3.1 & 0.0 & 0.6 & 0.8 & 1.2 & 2.3 & 0.3 & 0.8 & 0.3 & 0.0 & 0.0 & 0.7 & 1.7 & 100 \\
\hline Asa & F4 & $30-40$ & 43.2 & 15.1 & 27.7 & 2.2 & 3.4 & 0.0 & 0.5 & 0.8 & 1.5 & 2.4 & 0.4 & 0.5 & 0.2 & 0.3 & 0.0 & 0.1 & 1.6 & 100 \\
\hline Asa & F4 & $40-50$ & 42.2 & 15.8 & 27.8 & 2.1 & 3.4 & 0.0 & 0.5 & 0.7 & 1.6 & 2.6 & 0.4 & 0.5 & 0.2 & 0.3 & 0.0 & 0.0 & 1.8 & 100 \\
\hline Asa & F4 & $50-60$ & 42.6 & 15.7 & 27.7 & 2.1 & 3.3 & 0.0 & 0.5 & 0.8 & 1.5 & 2.2 & 0.5 & 0.7 & 0.4 & 0.3 & 0.0 & 0.0 & 1.7 & 100 \\
\hline Asa & F4 & $60-70$ & 43.6 & 14.4 & 27.1 & 2.2 & 3.4 & 0.0 & 0.6 & 0.7 & 1.8 & 2.7 & 0.2 & 0.5 & 0.2 & 0.6 & 0.0 & 0.0 & 1.9 & 100 \\
\hline Asa & F4 & $70-80$ & 43.2 & 15.2 & 26.6 & 2.3 & 3.7 & 0.0 & 0.6 & 0.9 & 1.8 & 2.1 & 0.5 & 0.8 & 0.4 & 0.5 & 0.0 & 0.0 & 1.6 & 100 \\
\hline Asa & F4 & $80-90$ & 40.7 & 15.6 & 28.6 & 2.4 & 3.1 & 0.0 & 0.4 & 0.8 & 1.8 & 3.6 & 0.3 & 0.1 & 0.2 & 0.7 & 0.0 & 0.0 & 1.6 & 100 \\
\hline Asa & F4 & $90-100$ & 42.8 & 15.2 & 28.9 & 1.9 & 3.1 & 0.0 & 0.5 & 0.8 & 1.4 & 2.6 & 0.5 & 0.1 & 0.2 & 0.3 & 0.0 & 0.0 & 1.7 & 100 \\
\hline
\end{tabular}

Flakaliden

\begin{tabular}{|c|c|c|c|c|c|c|c|c|c|c|c|c|c|c|c|c|c|c|c|c|}
\hline Site & Plot & Depth & Quartz & $\begin{array}{c}\text { K- } \\
\text { feldspar }\end{array}$ & $\begin{array}{l}\text { Plagio- } \\
\text { clase }\end{array}$ & $\begin{array}{c}\text { Amphi- } \\
\text { bole }\end{array}$ & $\begin{array}{c}\text { Epid- } \\
\text { ote }\end{array}$ & $\begin{array}{c}\text { Anat- } \\
\text { ase }\end{array}$ & $\begin{array}{c}\text { IIme- } \\
\text { nite }\end{array}$ & $\begin{array}{c}\text { Hema- } \\
\text { tite }\end{array}$ & $\begin{array}{c}\text { Chlorite } \\
\text { trioct. }\end{array}$ & $\begin{array}{l}\text { Mica } \\
\text { dioct. }\end{array}$ & $\begin{array}{l}\text { Mica } \\
\text { trioct. }\end{array}$ & $\begin{array}{l}\text { Verm. } \\
\text { dioct. }\end{array}$ & $\begin{array}{l}\text { Verm. } \\
\text { trioct. }\end{array}$ & $\begin{array}{l}\text { Hydro- } \\
\text { biotite }\end{array}$ & $\begin{array}{c}\text { Kaoli- } \\
\text { nite }\end{array}$ & $\begin{array}{l}\text { Im./ } \\
\text { Allo. }\end{array}$ & $\begin{array}{c}\text { Ferri- } \\
\text { hydrite }\end{array}$ & Total \\
\hline $\mathrm{F}$ & 11B & $0-10$ & 51.1 & 17.0 & 22.7 & 2.0 & 1.7 & 0.0 & 0.5 & 0.2 & 0.2 & 2.2 & 0.2 & 1.3 & 0.0 & 0.9 & 0.0 & 0.0 & 0.1 & 100 \\
\hline $\mathrm{F}$ & $11 \mathrm{~B}$ & $10-20$ & 47.1 & 14.2 & 22.7 & 2.9 & 2.0 & 0.0 & 0.4 & 0.2 & 0.6 & 3.2 & 0.0 & 1.0 & 0.0 & 0.8 & 0.0 & 2.9 & 2.0 & 100 \\
\hline $\mathrm{F}$ & $11 \mathrm{~B}$ & $20-30$ & 40.8 & 14.2 & 24.7 & 3.8 & 1.9 & 0.0 & 0.3 & 0.3 & 1.3 & 3.4 & 0.1 & 0.7 & 0.2 & 0.3 & 0.0 & 5.3 & 2.8 & 100 \\
\hline $\mathrm{F}$ & $11 \mathrm{~B}$ & $30-40$ & 39.3 & 13.6 & 25.5 & 4.7 & 2.5 & 0.0 & 0.2 & 0.2 & 1.9 & 3.8 & 0.1 & 0.7 & 0.2 & 0.5 & 0.0 & 3.4 & 3.3 & 100 \\
\hline $\mathrm{F}$ & $11 \mathrm{~B}$ & $40-50$ & 38.6 & 14.2 & 27.5 & 5.0 & 2.3 & 0.0 & 0.1 & 0.2 & 2.0 & 4.8 & 0.2 & 0.0 & 0.2 & 1.8 & 0.0 & 0.9 & 2.3 & 100 \\
\hline $\mathrm{F}$ & $11 \mathrm{~B}$ & $50-60$ & 40.6 & 13.5 & 25.9 & 5.2 & 2.6 & 0.0 & 0.2 & 0.3 & 2.0 & 4.5 & 0.7 & 0.0 & 0.2 & 1.4 & 0.0 & 0.8 & 2.1 & 100 \\
\hline $\mathrm{F}$ & 11B & $60-70$ & 40.8 & 14.4 & 26.3 & 2.7 & 1.4 & 0.0 & 0.1 & 0.1 & 1.7 & 4.1 & 1.4 & 0.3 & 0.2 & 2.1 & 0.0 & 2.7 & 1.9 & 100 \\
\hline $\mathrm{F}$ & $15 \mathrm{~A}$ & $0-10$ & 48.1 & 17.4 & 24.6 & 3.4 & 1.3 & 0.0 & 0.4 & 0.2 & 0.2 & 3.0 & 0.0 & 0.3 & 0.0 & 1.1 & 0.0 & 0.0 & 0.0 & 100 \\
\hline $\mathrm{F}$ & $15 \mathrm{~A}$ & $10-20$ & 42.1 & 14.2 & 23.7 & 6.0 & 1.6 & 0.0 & 0.3 & 0.2 & 1.0 & 2.4 & 0.0 & 1.4 & 0.1 & 0.8 & 0.0 & 3.7 & 2.6 & 100 \\
\hline $\mathrm{F}$ & $15 \mathrm{~A}$ & $20-30$ & 38.7 & 14.7 & 26.1 & 4.9 & 1.6 & 0.0 & 0.1 & 0.2 & 1.8 & 2.5 & 0.4 & 0.2 & 0.3 & 0.4 & 0.0 & 5.6 & 2.3 & 100 \\
\hline $\mathrm{F}$ & $15 \mathrm{~A}$ & $30-40$ & 38.4 & 18.2 & 32.3 & 2.6 & 1.4 & 0.0 & 0.1 & 0.2 & 1.3 & 2.2 & 0.8 & 0.2 & 0.0 & 0.8 & 0.0 & 0.6 & 0.8 & 100 \\
\hline $\mathrm{F}$ & $15 \mathrm{~A}$ & $40-50$ & 39.8 & 18.2 & 29.4 & 3.4 & 1.8 & 0.0 & 0.2 & 0.2 & 1.4 & 3.0 & 0.8 & 0.0 & 0.1 & 1.4 & 0.0 & 0.0 & 0.3 & 100 \\
\hline $\mathrm{F}$ & $15 \mathrm{~A}$ & $50-60$ & 40.8 & 16.7 & 28.7 & 3.8 & 2.0 & 0.0 & 0.2 & 0.2 & 1.8 & 3.2 & 0.8 & 0.0 & 0.0 & 0.8 & 0.0 & 0.0 & 1.0 & 100 \\
\hline $\mathrm{F}$ & $15 \mathrm{~A}$ & $60-70$ & 40.8 & 15.9 & 29.3 & 3.9 & 2.1 & 0.0 & 0.2 & 0.2 & 1.5 & 3.0 & 0.8 & 0.0 & 0.0 & 1.1 & 0.0 & 0.0 & 1.0 & 100 \\
\hline$F$ & $15 \mathrm{~A}$ & $70-80$ & 37.4 & 17.1 & 29.0 & 3.9 & 1.7 & 0.0 & 0.2 & 0.2 & 1.8 & 4.5 & 1.0 & 0.0 & 0.1 & 1.4 & 0.0 & 0.5 & 1.1 & 100 \\
\hline$F$ & $14 \mathrm{~B}$ & $0-10$ & 54.6 & 15.8 & 23.1 & 1.8 & 1.1 & 0.0 & 0.5 & 0.1 & 0.0 & 1.9 & 0.1 & 0.3 & 0.0 & 0.6 & 0.0 & 0.0 & 0.0 & 100 \\
\hline $\mathrm{F}$ & $14 B$ & $10-20$ & 51.4 & 16.9 & 23.4 & 2.1 & 1.6 & 0.0 & 0.3 & 0.2 & 0.1 & 2.7 & 0.1 & 0.2 & 0.0 & 0.8 & 0.0 & 0.0 & 0.0 & 100 \\
\hline $\mathrm{F}$ & $14 \mathrm{~B}$ & $20-30$ & 47.0 & 14.5 & 23.8 & 3.1 & 1.9 & 0.0 & 0.4 & 0.3 & 0.5 & 4.1 & 0.0 & 0.8 & 0.1 & 1.2 & 0.0 & 0.7 & 1.7 & 100 \\
\hline $\mathrm{F}$ & 14B & $30-40$ & 39.4 & 14.1 & 23.9 & 4.3 & 1.9 & 0.0 & 0.2 & 0.3 & 1.7 & 4.1 & 0.0 & 0.7 & 0.1 & 0.8 & 0.0 & 5.9 & 2.7 & 100 \\
\hline $\mathrm{F}$ & $14 B$ & $40-50$ & 40.4 & 13.6 & 27.0 & 4.4 & 2.4 & 0.0 & 0.2 & 0.2 & 1.9 & 3.7 & 0.2 & 0.5 & 0.3 & 1.1 & 0.0 & 1.8 & 2.3 & 100 \\
\hline $\mathrm{F}$ & $14 B$ & $50-60$ & 39.7 & 14.4 & 28.1 & 4.8 & 2.4 & 0.0 & 0.3 & 0.2 & 1.9 & 4.4 & 0.3 & 0.2 & 0.3 & 1.3 & 0.0 & 0.5 & 1.4 & 100 \\
\hline $\mathrm{F}$ & $14 B$ & $60-70$ & 40.7 & 14.3 & 26.6 & 5.1 & 2.6 & 0.0 & 0.3 & 0.2 & 2.0 & 4.5 & 0.2 & 0.1 & 0.3 & 1.5 & 0.0 & 0.1 & 1.5 & 100 \\
\hline $\mathrm{F}$ & $14 B$ & $70-80$ & 40.2 & 15.5 & 27.9 & 4.2 & 2.1 & 0.0 & 0.2 & 0.2 & 2.0 & 3.7 & 0.6 & 0.2 & 0.3 & 1.6 & 0.0 & 0.0 & 1.4 & 100.0 \\
\hline $\mathrm{F}$ & $14 B$ & $80-90$ & 40.1 & 15.5 & 27.7 & 4.2 & 1.9 & 0.0 & 0.2 & 0.2 & 1.8 & 4.0 & 1.2 & 0.0 & 0.1 & 1.6 & 0.0 & 0.0 & 1.5 & 100.0 \\
\hline $\mathrm{F}$ & $10 \mathrm{~B}$ & $0-10$ & 54.9 & 15.3 & 22.4 & 1.6 & 1.8 & 0.0 & 0.6 & 0.3 & 0.2 & 1.2 & 0.0 & 1.0 & 0.0 & 0.5 & 0.0 & 0.0 & 0.1 & 100.0 \\
\hline$F$ & $10 B$ & $10-20$ & 48.6 & 14.9 & 22.1 & 2.5 & 2.1 & 0.0 & 0.4 & 0.3 & 0.5 & 2.7 & 0.0 & 1.0 & 0.0 & 0.4 & 0.0 & 1.8 & 2.7 & 100.0 \\
\hline $\mathrm{F}$ & $10 \mathrm{~B}$ & $20-30$ & 38.5 & 13.5 & 23.2 & 4.4 & 2.3 & 0.0 & 0.2 & 0.3 & 1.4 & 3.8 & 0.0 & 0.0 & 0.3 & 0.4 & 0.0 & 8.1 & 3.6 & 100.0 \\
\hline $\mathrm{F}$ & $10 \mathrm{~B}$ & $30-40$ & 39.4 & 12.5 & 25.6 & 5.0 & 2.7 & 0.0 & 0.2 & 0.2 & 1.8 & 3.5 & 0.1 & 0.3 & 0.4 & 0.5 & 0.0 & 4.1 & 3.7 & 100.0 \\
\hline$F$ & $10 B$ & $40-50$ & No sample & & & & & & & & & & & & & & & & & \\
\hline $\mathrm{F}$ & $10 \mathrm{~B}$ & $50-60$ & 40.0 & 13.7 & 26.7 & 4.3 & 2.5 & 0.0 & 0.2 & 0.3 & 1.9 & 5.2 & 0.8 & 0.0 & 0.2 & 2.3 & 0.0 & 1.0 & 0.9 & 100.0 \\
\hline $\mathrm{F}$ & $10 \mathrm{~B}$ & $60-70$ & 40.5 & 14.3 & 25.9 & 2.8 & 1.6 & 0.0 & 0.1 & 0.1 & 2.0 & 4.2 & 1.5 & 0.3 & 0.3 & 2.3 & 0.0 & 2.3 & 1.8 & 100.0 \\
\hline $\mathrm{F}$ & $10 \mathrm{~B}$ & $70-80$ & 36.0 & 9.9 & 23.3 & 1.4 & 0.6 & 0.0 & 0.2 & 0.0 & 1.3 & 4.6 & 16.0 & 0.0 & 0.0 & 1.2 & 2.3 & 2.9 & 0.5 & 100.0 \\
\hline
\end{tabular}


Table S2. Fractional volumetric change (Vp)

\begin{tabular}{|c|c|c|c|}
\hline Site & Soil profile & Soil depth $(\mathrm{cm})$ & $V p$ \\
\hline Flakaliden & 10B3 & $0-10$ & 3.2 \\
\hline Flakaliden & 10B3 & $10-20$ & 1.8 \\
\hline Flakaliden & 10B3 & $20-30$ & 2.1 \\
\hline Flakaliden & 10B3 & $30-40$ & 1.2 \\
\hline Flakaliden & 10B3 & $40-50$ & 0.9 \\
\hline Flakaliden & 10B3 & $50-60$ & 0.5 \\
\hline Flakaliden & 10B3 & $60-70$ & 0.0 \\
\hline Flakaliden & 10B3 & $70-80$ & -0.3 \\
\hline Flakaliden & $11 \mathrm{~B}$ & $0-10$ & 1.6 \\
\hline Flakaliden & $11 B$ & $10-20$ & 0.4 \\
\hline Flakaliden & $11 B$ & $20-30$ & 0.4 \\
\hline Flakaliden & $11 \mathrm{~B}$ & $30-40$ & 0.1 \\
\hline Flakaliden & 11B & $40-50$ & 0.1 \\
\hline Flakaliden & $11 \mathrm{~B}$ & $50-60$ & 0.0 \\
\hline Flakaliden & $11 \mathrm{~B}$ & $60-70$ & -0.4 \\
\hline Flakaliden & 14B1 & $0-10$ & 2.0 \\
\hline Flakaliden & 14B1 & $10-20$ & 1.7 \\
\hline Flakaliden & 14B1 & $20-30$ & 1.8 \\
\hline Flakaliden & 14B1 & $30-40$ & 0.8 \\
\hline Flakaliden & 14B1 & $40-50$ & 0.2 \\
\hline Flakaliden & 14B1 & $50-60$ & 0.1 \\
\hline Flakaliden & 14B1 & $60-70$ & 0.0 \\
\hline Flakaliden & 14B1 & $70-80$ & 0.0 \\
\hline Flakaliden & 14B1 & $80-90$ & -0.2 \\
\hline Flakaliden & $15 A 3$ & $0-10$ & 1.5 \\
\hline Flakaliden & $15 A 3$ & $10-20$ & 1.0 \\
\hline Flakaliden & $15 A 3$ & $20-30$ & 0.5 \\
\hline Flakaliden & $15 A 3$ & $30-40$ & -0.2 \\
\hline Flakaliden & $15 A 3$ & $40-50$ & -0.4 \\
\hline Flakaliden & $15 \mathrm{~A} 3$ & $50-60$ & -0.3 \\
\hline Flakaliden & $15 A 3$ & $60-70$ & 0.0 \\
\hline Flakaliden & $15 \mathrm{A3}$ & $70-80$ & 0.4 \\
\hline Asa & F3 & $0-10$ & 0.4 \\
\hline Asa & F3 & $10-20$ & 0.3 \\
\hline Asa & F3 & $20-30$ & 0.2 \\
\hline Asa & F3 & $30-40$ & 0.2 \\
\hline Asa & F3 & $40-50$ & 0.1 \\
\hline Asa & F3 & $50-60$ & 0.2 \\
\hline Asa & F3 & $60-70$ & 0.0 \\
\hline Asa & F3 & $70-80$ & 0.0 \\
\hline Asa & F4 & $0-10$ & 1.3 \\
\hline Asa & F4 & $10-20$ & 1.3 \\
\hline Asa & F4 & $20-30$ & 0.5 \\
\hline Asa & F4 & $30-40$ & 0.2 \\
\hline Asa & F4 & $40-50$ & 0.4 \\
\hline Asa & F4 & $50-60$ & 0.1 \\
\hline Asa & F4 & $60-70$ & 0.0 \\
\hline Asa & F4 & $70-80$ & 0.1 \\
\hline Asa & F4 & $80-90$ & 0.0 \\
\hline
\end{tabular}




\begin{tabular}{|c|c|c|c|}
\hline Asa & F4 & $90-100$ & 0.0 \\
\hline Asa & K1II & $0-10$ & 2.4 \\
\hline Asa & K1II & 1020 & 2.2 \\
\hline Asa & K1II & $20-30$ & 1.3 \\
\hline Asa & K1II & $30-40$ & 1.5 \\
\hline Asa & K1II & $40-50$ & 1.0 \\
\hline Asa & K1II & $50-60$ & 0.6 \\
\hline Asa & K1II & $60-70$ & 0.3 \\
\hline Asa & K1II & $70-80$ & 0.1 \\
\hline Asa & K1II & $80-90$ & 0.0 \\
\hline Asa & K1II & $90-100$ & 0.4 \\
\hline Asa & K4 & $0-10$ & 2.6 \\
\hline Asa & K4 & $10-20$ & 0.7 \\
\hline Asa & K4 & $20-30$ & 0.8 \\
\hline Asa & K4 & $30-40$ & 0.5 \\
\hline Asa & K4 & $40-50$ & 0.4 \\
\hline Asa & K4 & $50-60$ & -0.1 \\
\hline Asa & K4 & $60-70$ & 0.0 \\
\hline Asa & K4 & $70-80$ & -0.1 \\
\hline Asa & K4 & $80-90$ & 0.0 \\
\hline Asa & K4 & $90-100$ & -0.1 \\
\hline
\end{tabular}


Table S3. Soil physical input data to PROFILE (i.e. Soil bulk density and exposed mineral surface area)

Exposed mineral

Soil depth Soil bulk density surface area $\left(\mathrm{m}^{2}\right.$ Moisture content Dissolved organic Aluminium solubility Soil solution $\mathrm{CO}_{2}$

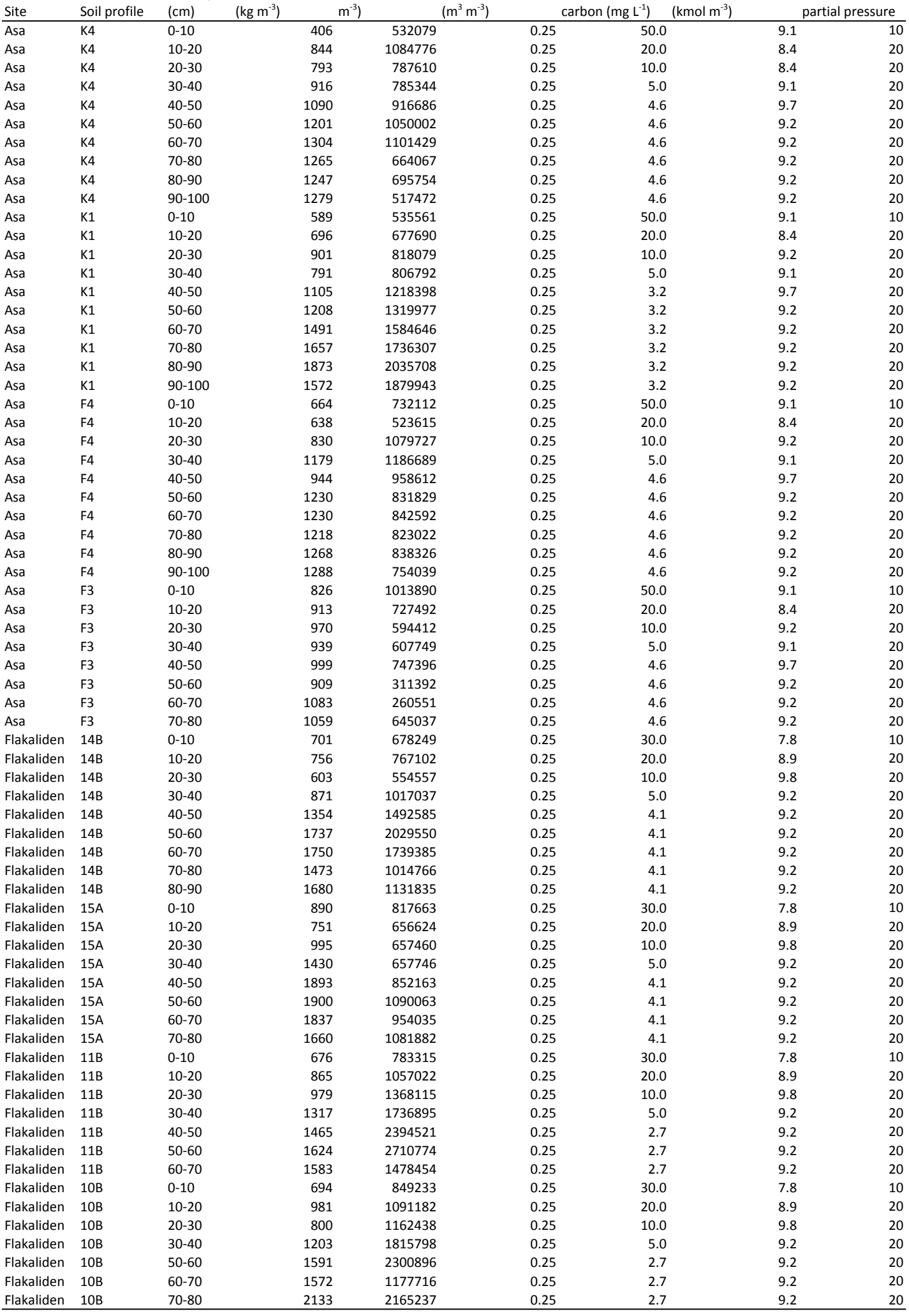

